\title{
Unilateral asterixis due to a lesion of the ventrolateral thalamus
}

\author{
R Stell, S Davis, W M Carroll
}

\begin{abstract}
A case of unilateral asterixis in a man with a focal ischaemic lesion of the contralateral ventral thalamus is presented. Atypically, the movements were present at rest and had a pattern of activation that resulted in an initial misdiagnosis of epilepsia partialis continua. This case emphasises the importance of electromyographic analysis in establishing the correct diagnosis of involuntary movements before starting specific treatment.
\end{abstract}

(F Neurol Neurosurg Psychiatry 1994;57:1 16-118)

The term asterixis was first used by Adams and Foley ${ }^{1}$ to describe the asynchronous jerky twitching movements seen in association with the flapping tremor of hepatic encephalopathy. Asterixis may also be seen with other causes of metabolic encephalopathy including renal and respiratory failure, drug intoxication, and electrolyte imbalance. In such cases the asterixis is usually bilateral, although often asymmetric, and is accompanied by an altered level of consciousness. These features, associated with a diffuse EEG slowing, have led to the perception that asterixis represents a non-specific and non-localising abnormality of the motor system. In contrast to bilateral asterixis, unilateral cases are relatively uncommon and have been associated with a variety of focal cerebral lesions. ${ }^{23}$ Although asterixis was at first thought to result from severe loss of joint position sense, clinical experience has suggested that this is not the case in many instances.

In this paper we describe a case of unilateral asterixis in a patient with a focal lesion of the contralateral thalamus in whom no significant sensory deficit was evident at the time. The unilateral nature of the movements and their unusual character had initially led to a misdiagnosis of epilepsia partialis continua. This case provides further evidence that unilateral asterixis may result from a focal structural lesion of the ventrolateral thalamus, and highlights the difficulty that may arise in making the diagnosis without the aid of electromyographic studies.

\section{Case report}

A 73-year-old right-handed man presented with a two-week history of left-sided paraes- thesiae and weakness of the face, arm, and leg which recovered incompletely over the next week. He was found to be hypertensive and was commenced on enalapril. Ten days later there was sudden worsening of the left arm and leg weakness such that he was unable to walk. There were no sensory symptoms at that time. There was a past history of pulmonary tuberculosis and empyema. He had been a heavy smoker for three years before admission and consumed $20 \mathrm{~g}$ alcohol per day.

On examination he was alert and orientated and bedside testing of higher cerebral function was normal. The uncorrected visual acuities were $6 / 18$ bilaterally. There was visual neglect in the right hemifield and arteriolar attenuation on fundal examination. Ocular motor function was normal apart from saccadic intrusion into horizontal pursuit more marked to the right than the left. There was a left upper motor neuron facial weakness, weakness of head turning to the left, and hypertonia, hyperreflexia, and a pyramidal pattern of weakness in the left arm. Mild sensory neglect was present in the left hand but there was no impairment of either higher order discriminatory function or the primary sensory modalities. There was bilateral hyperreflexia hypertonia and mild left-sided weakness in the legs. Occasional errors of joint position sense were noted in the toes and vibration sensation was impaired to the knees bilaterally. The plantar response was extensor on the left and flexor on the right.

In the left arm, involuntary semi-rhythmic abduction and flexion movements of the fingers and flexion of the wrist were present regardless of the posture of the limb. These movements, which were not stimulus sensitive and persisted into sleep, were thought to be typical of epilepsia partialis continua. In contrast, with the left wrist held in extension and the arms pronated, rhythmic loss of posture resulted in wrist flexion movements typical of those seen in asterixis. These were associated with the aforementioned finger movements.

Routine biochemistry was normal, as were fasting lipids, blood glucose, and venereal disease estimations. The haemoglobin was reduced at $11.8 \mathrm{~g} / \mathrm{dl}$ with normal red cell indices; the white blood cell count was normal but hypersegmented neutrophils were present. Serum vitamin B-12 was less than 50 $\mathrm{ng} / 1$ (normal range 200-1000), and serum 
Figure 1 A T2 weighted MRI scan showing the right thalamic lesion involving the lateralventral group and pulvinar $(A)$, extending into the immediate periventricular white matter of the corona radiata superiorly (B). Subcortical infarction of the right frontal lobe and presumed deep white matter ischaemic changes in the left parietal lobe are also shown.

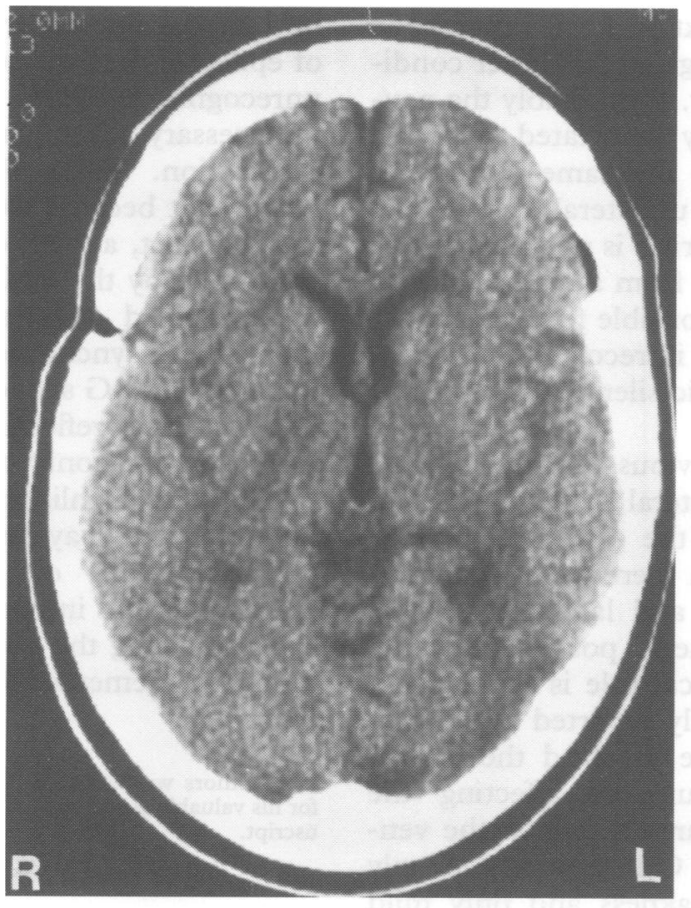

(A)

antibodies to intrinsic factor were detected. The CSF examination was normal. The chest radiograph showed left pleural calcification consistent with healed tuberculous empyema. The EEG was abnormal with focal slow wave activity in the right anterior temporal region on a generally slow background but no paroxysmal or epileptiform discharges were present. A cerebral CT scan performed 14 days after the onset of limb weakness demonstrated regions of low density in the right ventral and lateral thalamus and in the right precentral frontal cortex. After contrast injection, the right frontal cortical lesion developed a surrounding area of enhancement (luxury perfusion), confirming its recent occurrence. Cranial magnetic resonance imaging performed 19 days after the onset of weakness (fig 1a), demonstrated hyperinten-

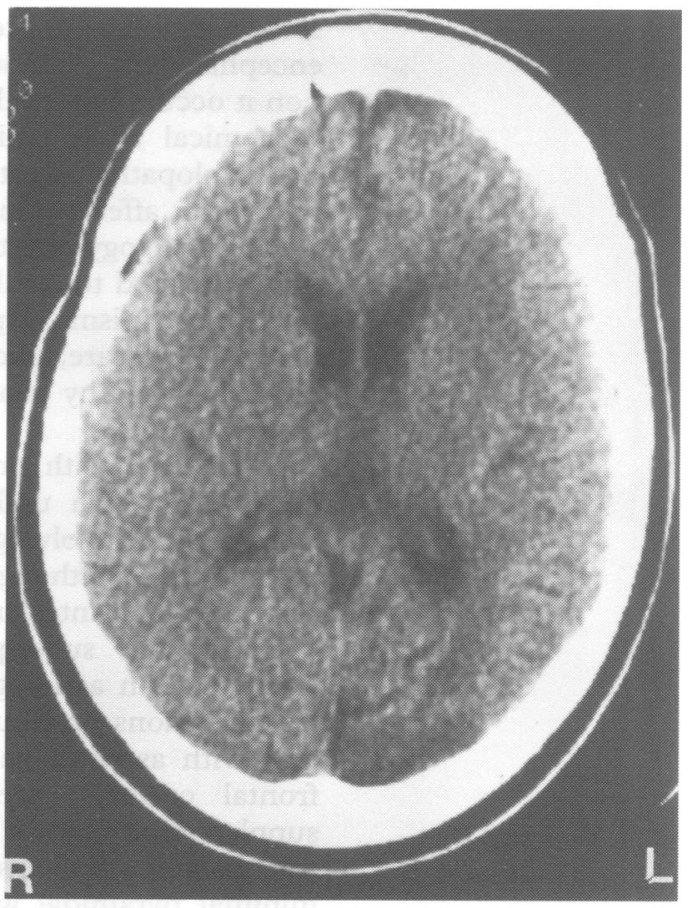

(B)

sity on $\mathrm{T} 2$ weighted images in the right lateral and ventral thalamus and pulvinar extending into the periventricular white matter and the adjacent corona radiata (fig $1 \mathrm{~b}$ ). Zones of high signal intensity were also evident in the subcortical white matter of the right posterior frontal lobe and in the left parietal white matter. A carotid duplex doppler study demonstrated complete occlusion of the right internal carotid artery.

Electromyographic (EMG) activity was recorded simultaneously from the left forearm flexor and extensor muscles using $10 \mathrm{~mm}$ gold cup surface electrodes and a Medelec Mystro. With the arms extended and pronated, irregular bursts of muscle activity were interspersed with periods of relative silence lasting approximately $20 \mathrm{~ms}$, the latter coinciding with the flexion movements of the fingers and wrist (fig 2).

\section{Discussion}

In 1964 Leavitt and Tyler $^{2}$ noted unilateral asterixis in the occasional patient with focal cerebral lesions of the thalamus or parietal cortex resulting in severe proprioceptive loss. They postulated a 'high level' defect in the continuous integration of joint position and other sensory information as the cause for asterixis. Young and Shahani ${ }^{3}$ described unilateral asterixis with focal lesions of the contralateral thalamus, parietal lobe, internal capsule, or medial frontal cortex in patients without proprioceptive loss, weakness, or clumsiness in the affected limb. They suggested that unilateral asterixis was of localising value and could occur in patients without associated stupor or signs of diffuse cerebral dysfunction. Although asterixis produced by unilateral focal CNS lesions is uncommon, its appearance is indistinguishable from that
Figure 2 Electromyographic (EMG) activity recorded from the forearm extensor (EDC) and flexor (FDS) muscles. The output of an accelerometer placed on the dorsum of the left wrist is shown in the top tracing. Flexion is represented by an upward deflection of the trace and extension by a downward deflection. The arrow indicates the point at which EMG activity transiently ceases. This is followed by wrist flexionlextension after an interval of 70-80 ms; the initial movements occurred during this period of EMG silence. 
seen in the context of a metabolic encephalopathy, although in this latter condition it occurs bilaterally. Presumably the neurochemical abnormality associated with the encephalopathy affects the same regions of the brain affected in unilateral cases. The pathophysiology of asterixis is not known, but it is presumed to result from a disturbance of those mechanisms responsible for the maintenance of posture, and is recognised electrophysiologically by phasic silent periods in the ongoing EMG.

In keeping with previous reported cases, ${ }^{4}$ our patient with unilateral asterixis had an initial lesion involving the contralateral ventral thalamus, although there was also extension into the anterior and lateral thalamus. Whether the subsequent posterior frontal lesion had an aetiological role is speculative; frontal lesions previously reported in association with asterixis have involved the medial frontal cortex, ${ }^{35}$ presumably affecting the supplementary motor area to which the ventral thalamus projects. Our patient had only minimal pyramidal weakness and only mild sensory neglect supporting the view of Young and Shahani ${ }^{6}$ that asterixis is not caused by deafferentation of the affected limb. It could be postulated that a lesion of the ventrolateral 'motor' thalamus or its immediate connections, or both, might result in intermittent inhibition or failure of activation of the supplementary motor area, producing the characteristic EMG 'silent periods' and clinical picture of asterixis.

Of note in our patient was the finding that the movements of the fingers and hand were present even with the limb at rest, and were initially considered erroneously to be a form of epilepsia partialis continua, a fact which, if unrecognised, may have resulted in the unnecessary administration of anticonvulsant medication. When the limb was elevated, however it became clear that the movements were, in fact, a form of asterixis and this was confirmed by the EMG studies which clearly demonstrated that the involuntary movements were synchronous with loss of muscle tonus and EMG silence; the movement of the fingers simply reflecting the net result of the inhibition on agonist-antagonist muscle pairs.

This case highlights the potential diagnostic errors that may result from a purely clinical assessment of such movements and emphasises the importance of EMG analysis in establishing the correct diagnosis of involuntary movements before beginning specific treatment.

The authors would like to thank Professor Frank Mastaglia for his valuable comments during the preparation of this manuscript.

1 Adams RD, Foley JM. The neurological changes in the more common types of severe liver disease. Trans $\mathrm{Am}$ Neurol Assoc 1949;74:217-9.

2 Leavitt S, Tyler HR. Studies in asterixis. Arch Neurol 1964;10:360-8.

3 Young RR, Shahani BT. Anticonvulsant asterixis. Electroencephalogr Clin Neurophysiol 1973;34:760a.

4 Young RR, Shahani BT, Kjellberg RN. Unilateral asterixis produced by a discrete CNS lesion. Trans Am ixis produced by a discrete
Neurol Assoc $1976 ; 101: 306-7$.

5 Santamaria-Cano J, Graus-Ribas F, Martinez-Matos J, Rubio-Borrero F, Arbizu-Urdiain T, Peres-Serra J. Asterixis en lesiones focales del sistema nervioso central. Rev Clin Esp 1983;168:37-9.

6 Young RR, Shahani BT. Asterixis: one type of negative myoclonus In: Fahn S, Marsden CD, Van Woert MH, eds. Advances in Neurology, vol 43: Myoclonus. New York: Raven Press, 1986:137-56. 


\title{
Unilateral asterixis due to a lesion of the ventrolateral thalamus ${ }^{\star}$
}

\author{
R Stell, S Davis, W M Carroll
}

\begin{abstract}
A case of unilateral asterixis in a man with a focal ischaemic lesion of the contralateral ventral thalamus is presented. Atypically, the movements were present at rest and had a pattern of activation that resulted in an initial misdiagnosis of epilepsia partialis continua. This case emphasises the importance of electromyographic analysis in establishing the correct diagnosis of involuntary movements before starting specific treatment.
\end{abstract}

$(\mathcal{F}$ Neurol Neurosurg Psychiatry 1994;57:878-880)

The term asterixis was first used by Adams and Foley ${ }^{1}$ to describe the asynchronous jerky twitching movements seen in association with the flapping tremor of hepatic encephalopathy. Asterixis may also be seen with other causes of metabolic encephalopathy including renal and respiratory failure, drug intoxication, and electrolyte imbalance. In such cases the asterixis is usually bilateral, although often asymmetric, and is accompanied by an altered level of consciousness. These features, associated with a diffuse EEG slowing, have led to the perception that asterixis represents a non-specific and non-localising abnormality of the motor system. In contrast to bilateral asterixis, unilateral cases are relatively uncommon and have been associated with a variety of focal cerebral lesions. ${ }^{23}$ Although asterixis was at first thought to result from severe loss of joint position sense, clinical experience has suggested that this is not the case in many instances.

In this paper we describe a case of unilateral asterixis in a patient with a focal lesion of the contralateral thalamus in whom no significant sensory deficit was evident at the time. The unilateral nature of the movements and their unusual character had initially led to a misdiagnosis of epilepsia partialis continua. This case provides further evidence that unilateral asterixis may result from a focal structural lesion of the ventrolateral thalamus, and highlights the difficulty that may arise in making the diagnosis without the aid of electromyographic studies.

\section{Case report}

A 73-year-old right-handed man presented with a two-week history of left-sided paraes- thesiae and weakness of the face, arm, and leg which recovered incompletely over the next week. He was found to be hypertensive and was commenced on enalapril. Ten days later there was sudden worsening of the left arm and leg weakness such that he was unable to walk. There were no sensory symptoms at that time. There was a past history of pulmonary tuberculosis and empyema. He had been a heavy smoker for three years before admission and consumed $20 \mathrm{~g}$ alcohol per day.

On examination he was alert and orientated and bedside testing of higher cerebral function was normal. The uncorrected visual acuities were $6 / 18$ bilaterally. There was visual neglect in the right hemifield and arteriolar attenuation on fundal examination. Ocular motor function was normal apart from saccadic intrusion into horizontal pursuit more marked to the right than the left. There was a left upper motor neuron facial weakness, weakness of head turning to the left, and hypertonia, hyperreflexia, and a pyramidal pattern of weakness in the left arm. Mild sensory neglect was present in the left hand but there was no impairment of either higher order discriminatory function or the primary sensory modalities. There was bilateral hyperreflexia hypertonia and mild left-sided weakness in the legs. Occasional errors of joint position sense were noted in the toes and vibration sensation was impaired to the knees bilaterally. The plantar response was extensor on the left and flexor on the right.

In the left arm, involuntary semi-rhythmic abduction and flexion movements of the fingers and flexion of the wrist were present regardless of the posture of the limb. These movements, which were not stimulus sensitive and persisted into sleep, were thought to be typical of epilepsia partialis continua. In contrast, with the left wrist held in extension and the arms pronated, rhythmic loss of posture resulted in wrist flexion movements typical of those seen in asterixis. These were associated with the aforementioned finger movements.

Routine biochemistry was normal, as were fasting lipids, blood glucose, and venereal disease estimations. The haemoglobin was reduced at $11.8 \mathrm{~g} / \mathrm{dl}$ with normal red cell indices; the white blood cell count was normal but hypersegmented neutrophils were present. Serum vitamin B-12 was less than 50 ng/1 (normal range 200-1000), and serum 
Figure $1 A T 2$ weighted MRI scan showing the involving the lateral ventral group cnd pulvinar $(A)$, extending into the immediate periventricular white matter of the corona radiata superiorly $(B)$. Subcortical infarction of presumed deep white matter ischaemic changes in the left parietal lobe are also shown. right thalamic lesion the right frontal lobe and

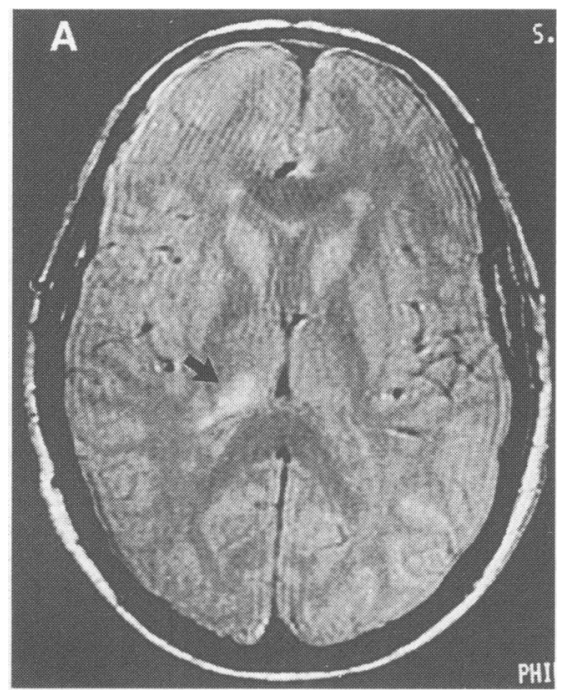

antibodies to intrinsic factor were detected. The CSF examination was normal. The chest radiograph showed left pleural calcification consistent with healed tuberculous empyema. The EEG was abnormal with focal slow wave activity in the right anterior temporal region on a generally slow background but no paroxysmal or epileptiform discharges were present. A cerebral CT scan performed 14 days after the onset of limb weakness demonstrated regions of low density in the right ventral and lateral thalamus and in the right precentral frontal cortex. After contrast injection, the right frontal cortical lesion developed a surrounding area of enhancement (luxury perfusion), confirming its recent occurrence. Cranial magnetic resonance imaging performed 19 days after the onset of weakness (fig 1a), demonstrated hyperintensity on T2 weighted images in the right lateral and ventral thalamus and pulvinar extending into the periventricular white matter and the adjacent corona radiata (fig $1 \mathrm{~b}$ ). Zones of high signal intensity were also evident in the subcortical white matter of the right posterior frontal lobe and in the left parietal white

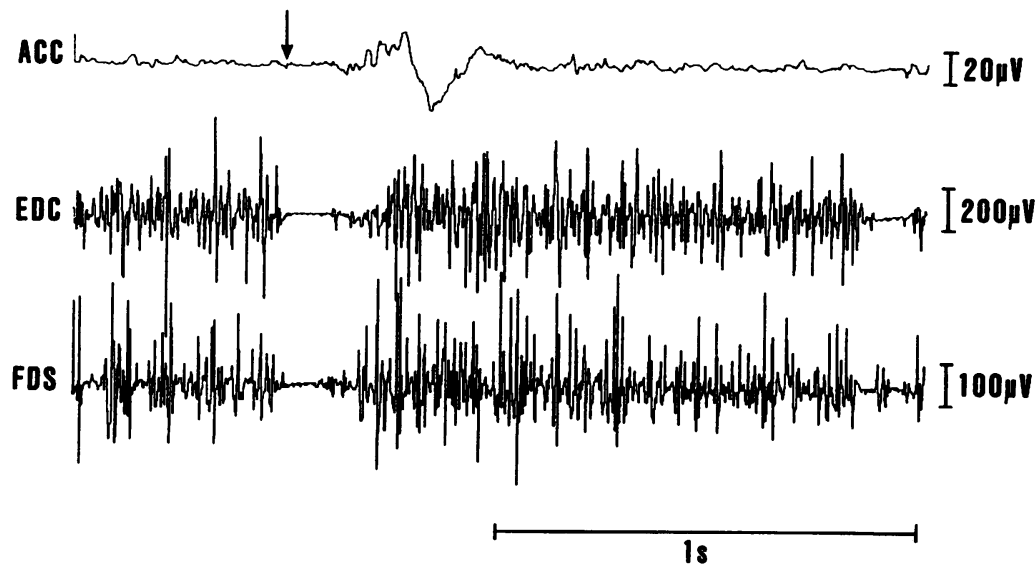

Figure 2 Electromyographic (EMG) activity recorded from the forearm extensor (EDC) and flexor (FDS) muscles. The output of an accelerometer placed on the dorsum of the left wrist is shown in the top tracing. Flexion is represented by an upward deflection of the trace and extension by a downward deflection. The arrow indicates the point at which EMG activity transiently ceases. This is followed by wrist flexion/extension after an interval of 70-80 ms; the initial movements occurred during this period of EMG silence.

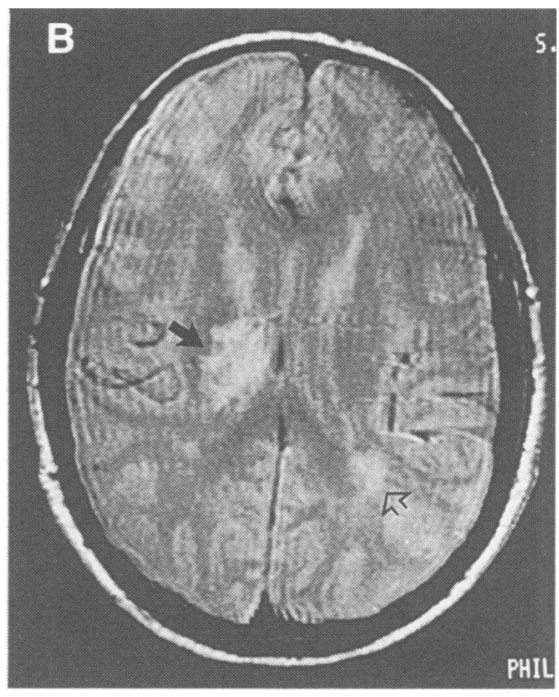

matter. A carotid duplex doppler study demonstrated complete occlusion of the right internal carotid artery.

Electromyographic (EMG) activity was recorded simultaneously from the left forearm flexor and extensor muscles using $10 \mathrm{~mm}$ gold cup surface electrodes and a Medelec Mystro. With the arms extended and pronated, irregular bursts of muscle activity were interspersed with periods of relative silence lasting approximately $20 \mathrm{~ms}$, the latter coinciding with the flexion movements of the fingers and wrist (fig 2).

\section{Discussion}

In 1964 Leavitt and Tyler $^{2}$ noted unilateral asterixis in the occasional patient with focal cerebral lesions of the thalamus or parietal cortex resulting in severe proprioceptive loss. They postulated a 'high level' defect in the continuous integration of joint position and other sensory information as the cause for asterixis. Young and Shahani ${ }^{3}$ described unilateral asterixis with focal lesions of the contralateral thalamus, parietal lobe, internal capsule, or medial frontal cortex in patients without proprioceptive loss, weakness, or clumsiness in the affected limb. They suggested that unilateral asterixis was of localising value and could occur in patients without associated stupor or signs of diffuse cerebral dysfunction. Although asterixis produced by unilateral focal CNS lesions is uncommon, its appearance is indistinguishable from that seen in the context of a metabolic encephalopathy, although in this latter condition it occurs bilaterally. Presumably the neurochemical abnormality associated with the encephalopathy affects the same regions of the brain affected in unilateral cases. The pathophysiology of asterixis is not known, but it is presumed to result from a disturbance of those mechanisms responsible for the maintenance of posture, and is recognised electrophysiologically by phasic silent periods in the ongoing EMG.

In keeping with previous reported cases, ${ }^{4}$ our patient with unilateral asterixis had an 
initial lesion involving the contralateral ventral thalamus, although there was also extension into the anterior and lateral thalamus. Whether the subsequent posterior frontal lesion had an aetiological role is speculative; frontal lesions previously reported in association with asterixis have involved the medial frontal cortex, ${ }^{35}$ presumably affecting the supplementary motor area to which the ventral thalamus projects. Our patient had only minimal pyramidal weakness and only mild sensory neglect supporting the view of Young and Shahani ${ }^{6}$ that asterixis is not caused by deafferentation of the affected limb. It could be postulated that a lesion of the ventrolateral 'motor' thalamus or its immediate connections, or both, might result in intermittent inhibition or failure of activation of the supplementary motor area, producing the characteristic EMG "silent periods" and clinical picture of asterixis.

Of note in our patient was the finding that the movements of the fingers and hand were present even with the limb at rest, and were initially considered erroneously to be a form of epilepsia partialis continua, a fact which, if unrecognised, may have resulted in the unnecessary administration of anticonvulsant medication. When the limb was elevated, however it became clear that the movements were, in fact, a form of asterixis and this was confirmed by the EMG studies which clearly demonstrated that the involuntary movements were synchronous with loss of muscle tonus and EMG silence; the movement of the fingers simply reflecting the net result of the inhibition on agonist-antagonist muscle pairs.

This case highlights the potential diagnostic errors that may result from a purely clinical assessment of such movements and emphasises the importance of EMG analysis in establishing the correct diagnosis of involuntary movements before beginning specific treatment.

The authors would like to thank Professor Frank Mastaglia for his valuable comments during the preparation of this manuscript.

1 Adams RD, Foley JM. The neurological changes in the more common types of severe liver disease. Trans $A m$ Neurol Assoc 1949;74:217-9.

2 Leavitt S, Tyler HR. Studies in asterixis. Arch Neurol 1964;10:360-8

3 Young RR, Shahani BT. Anticonvulsant asterixis. Electroencephalogr Clin Neurophysiol 1973;34:760a.

4 Young RR, Shahani BT, Kjellberg RN. Unilateral asterixis produced by a discrete CNS lesion. Trans Am Neurol Assoc 1976;101:306-7.

5 Santamaria-Cano J, Graus-Ribas F, Martinez-Matos J, Rubio-Borrero F, Arbizu-Urdiain T, Peres-Serra J. Rubio-Borrero F, Arbizu-Urdain T, Peres-Serra J. Rev Clin Esp 1983;168:37-9.

6 Young RR, Shahani BT. Asterixis: one type of negative myoclonus In: Fahn S, Marsden CD, Van Woert MH, eds. Advances in Neurology, vol 43: Myoclonus. New eds. Advances in Neurology, vol
York: Raven Press, 1986:137-56. 